\title{
Sorafenib-induced Posterior Reversible Encephalopathy Syndrome in a Child With FLT3-ITD-positive Acute Myeloid Leukemia
}

\author{
Betul Tavil, MD,* Fatma Isgandarova, MD, $\dagger$ Turan Bayhan, MD, $\dagger$ \\ Sule Unal, MD,* Baris Kuskonmaz, MD, * Fatma Gumruk, MD,* \\ and Mualla Cetin, MD*
}

\begin{abstract}
Background: Posterior reversible encephalopathy syndrome (PRES) is a clinical and radiologic condition characterized by headache, seizures, impaired vision, acute hypertension, and typical cranial MRI findings.

Observation: A 10-year-old boy with FLT3-ITD-positive acute myelogenous leukemia who developed PRES during sorafenib treatment has been presented here. In English literature, there are 2 adult patients with metastatic cholangiocarcinoma or hepatocellular carcinoma who developed PRES under sorafenib treatment. Our patient is the first pediatric case with the diagnosis of acute myelogenous leukemia who developed PRES that might be attributed to sorafenib use.
\end{abstract}

Conclusions: Thus, PRES might be a rare, potentially serious, but manageable, side effect of sorafenib that should be kept in mind by pediatric hematologists and oncologists.

Key Words: sorafenib, acute myeloid leukemia, children

(J Pediatr Hematol Oncol 2016;38:240-242)

$P_{a}$ osterior reversible encephalopathy syndrome (PRES) is a clinical and radiologic condition characterized by headache, seizures, impaired vision, acute hypertension, and typical magnetic resonance imaging (MRI) findings. Symmetric and reversible bilateral gray and white matter edema in the posterior regions of the cerebral hemispheres is the typical finding on brain MRI. Immunosuppressive and cytotoxic agents are common triggers for the development of PRES in children and adults. ${ }^{1}$

Sorafenib is an oral multiple tyrosine kinase inhibitor. The main targets of sorafenib are receptor tyrosine kinase pathways frequently deregulated in cancer, such as the RafRas, vascular endothelial growth factor (VEGF), and FMS-like tyrosine kinase 3 (FLT3) pathways. Sorafenib was approved by the FDA for the treatment of advanced renal cell cancer, hepatocellular cancer, and thyroid cancer. ${ }^{2}$ FMS-like tyrosine kinase 3-internal tandem duplication (FLT3-ITD) activating mutations are seen in leukemic cells of approximately $30 \%$ of adults and $15 \%$ of children

Received for publication February 23, 2015; accepted January 18, 2016. From the *Pediatric Hematology Unit; and †Hacettepe University Faculty of Medicine, Ihsan Dogramaci Children's Hospital, Sihhiye, Ankara, Turkey.

The authors declare no conflict of interest.

Reprints: Betul Tavil, Pediatric Hematology Unit, Hacettepe University Faculty of Medicine, Ihsan Dogramaci Children's Hospital, Sihhiye 06100, Ankara, Turkey (e-mail: tavil@ hacettepe.edu.tr) Copyright (C) 2016 Wolters Kluwer Health, Inc. All rights reserved. with acute myelogenous leukemia (AML) at diagnosis. ${ }^{3}$ FLT3-ITD-positive AML cases are associated with a poor prognosis. ${ }^{4}$ The small-molecule tyrosine kinase inhibitors, including sorafenib, showed potent activity against leukemic cells with FLT3-ITD mutations. ${ }^{3,4}$

A 10-year-old boy with FLT3-ITD-positive AML, who developed PRES during sorafenib treatment, is described here. In the English literature, there are only 2 adult patients with metastatic cholangiocarcinoma and hepatocellular carcinoma who developed PRES during sorafenib treatment. Our patient is the first pediatric case with the diagnosis of AML who developed PRES, which might be attributed to sorafenib use.

\section{CASE REPORT}

A 10-year-old boy was admitted to our hospital with chest pain. His body weight was $28 \mathrm{~kg}$ ( 25 percentile) and height was $128 \mathrm{~cm}$ (25 percentile). Physical examination revealed hepatosplenomegaly (liver $2 \mathrm{~cm}$ and spleen $4 \mathrm{~cm}$ below the costal margin). The complete blood count showed a hemoglobin level of $7.5 \mathrm{~g} / \mathrm{dL}$, a leukocyte count of $84.6 \times 10^{9} / \mathrm{L}$, and a platelet count of $28 \times 10^{9} / \mathrm{L}$. Myeloblasts were seen on peripheral blood and bone marrow aspiration smears. Flow cytometry analysis of the bone marrow revealed CD13, CD33, CD34, CD117, and MPO positivity, which was suggestive of AML. There was no extramedullary involvement at diagnosis. AML BFM 2004 chemotherapy protocol was started after diagnosis.

The AML BFM 2004 induction protocol (AIE block) included cytosine arabinoside (Ara-C), continuous intravenous infusion for 48 hours $\left(100 \mathrm{mg} / \mathrm{m}^{2} / \mathrm{d}\right.$; days 1 to 3$)$, followed by $100 \mathrm{mg} / \mathrm{m}^{2} /$ dose at 12 -hour intervals for 6 days (days 3 to 8 ), etoposide $\left(150 \mathrm{mg} / \mathrm{m}^{2} / \mathrm{d}\right.$; days $\left.6-8\right)$, idarubicin $\left(12 \mathrm{mg} / \mathrm{m}^{2} / \mathrm{d}\right.$; days 3,5 , and 7), and intrathecal Ara-C (40 mg; days 1 and 8$)$. Consolidation (HAM block) included high-dose Ara-C $\left(3000 \mathrm{mg} / \mathrm{m}^{2} /\right.$ dose $)$ every 12 hours for 6 doses (days 1 to 3$)$, mitoxantrone $\left(10 \mathrm{mg} / \mathrm{m}^{2} / \mathrm{d}\right.$; days 3 and 4), and intrathecal Ara-C on day 1. Unfortunately, remission was not achieved. However, cytogenetic studies revealed an FLT3ITD mutation. A donor search for hematopoietic stem cell transplantation was started, and an HLA-identical sister was identified The next induction attempt used the FLAG-IDA chemotherapy regimen including fludarabine $\left(30 \mathrm{mg} / \mathrm{m}^{2} / \mathrm{d}\right.$; days 1 to 4$)$, Ara-C $\left(2000 \mathrm{mg} / \mathrm{m}^{2} / \mathrm{d}\right.$; days 1 to 4$)$, and idarubicin $\left(12 \mathrm{mg} / \mathrm{m}^{2} / \mathrm{d}\right.$; days 2 to 4). However, myeloblasts appreciated on the peripheral blood smear after 1 month of FLAG-IDA regimen. The leukocyte count tended to increase rapidly. A salvage chemotherapy regimen including clofarabine $\left(40 \mathrm{mg} / \mathrm{m}^{2} / \mathrm{d}\right.$; days 1 to 5$)$, Ara-C $(1000 \mathrm{mg}$ $\mathrm{m}^{2} / \mathrm{d}$; days 1 to 5 ), and intrathecal Ara-C $(40 \mathrm{mg})$ on day 1 , followed by sorafenib (100 mg twice daily for $28 \mathrm{~d}$ ), was initiated. Sorafenib was added after the administration of 5 days of clofarabine and Ara-C.

However, the patient developed a headache and experienced a generalized tonic-clonic seizure after 14 days of sorafenib treatment. His blood pressure (BP) was normal $(<90$ percentile $[90$ percentile: 
$74 / 114 \mathrm{~mm} \mathrm{Hg}$ ] according to his age and height percentile) before and during sorafenib treatment. However, his BP was $125 / 80 \mathrm{~mm} \mathrm{Hg}$ during seizure (systolic $\mathrm{BP} \geq 99$ percentile and diastolic $\mathrm{BP} \geq 95$ according to his age and height percentile) ${ }^{5} \mathrm{He}$ had no focal neurological deficits. His electrolytes, blood urea nitrogen, and creatinine values were normal, and he had no proteinuria. Intravenous midazolam treatment at a dose of $0.1 \mathrm{mg} / \mathrm{kg}$ was administered during the seizure. After seizure was stopped with midazolam treatment, phenytoin treatment (loading dose of $10 \mathrm{mg} / \mathrm{kg}$ in 2 doses and maintenance dose of 6 to $7 \mathrm{mg} / \mathrm{kg}$ in 2 divided doses) was also given to the patient. Brain MRI obtained immediately after the onset of symptoms showed confluent hyperintense $\mathrm{T} 2$ signal involving subcortical white matter of the parietooccipital lobes consistent with PRES (Figs. 1A, B). Sorafenib was discontinued and levetiracetam (an antiepileptic drug) was started after the diagnosis of PRES. All the symptoms were resolved after 24 hours of discontinuation of sorafenib. However, the patient subsequently died with resistant disease. He was not rechallenged with sorafenib treatment.

\section{DISCUSSION}

PRES, initially reported by Hinchey et $\mathrm{al}^{6}$ in 1996 , is a clinical and radiologic condition characterized by headache, seizures, impaired vision, acute hypertension, and typical brain MRI findings. Pathogenesis of PRES begins with the onset of hypertension and/or damage to the capillary endothelium in the brain leading to the capillary leak and vasogenic edema. This syndrome has been described in association with different clinical conditions, including collagen vascular disorders (systemic lupus erythematosus, polyarteritis nodose, Behcet disease), thrombotic thrombocytopenic purpura, eclampsia, essential hypertension, and exposure to immunosuppressive and cytotoxic drugs such as cyclosporine A, tacrolimus, interferon- $\alpha$, cisplatin, cytarabine, IV immunoglobulins, L-asparaginase, bevacizumab (antiangiogenic agent), sorafenib, and sunitinib (tyrosine kinase inhibitors). ${ }^{7-11}$

In the English literature, there are several patients who developed PRES after bevacizumab treatment. Two of the patients were children. ${ }^{12,13}$ However, there are only 3 and 2 adult patients who developed PRES after sunitinib and sorafenib treatment, respectively. ${ }^{14}$ One of them was a 49-year-old woman with metastatic cholangiocarcinoma. After 4 months of sorafenib ( $400 \mathrm{mg}$ twice daily), she developed severe headache, increased BP, and sudden loss of vision, followed by a generalized tonic-clonic seizure and loss of consciousness. Her brain MRI revealed typical signal changes in the posterior cerebral white matter, consistent with PRES. After sorafenib was stopped, she recovered from her clinical condition and she was discharged from the hospital on the third day of cessation of the drug. ${ }^{8}$

The other patient was a 58-year-old man with hepatocellular carcinoma. After 1 week of sorafenib $(400 \mathrm{mg}$ twice daily), he developed headache and blurred vision. His clinical findings were resolved within 1 week after sorafenib treatment was discontinued. Then, sorafenib treatment was restarted. However, he again complained of dizziness, hallucinations, hypersalivation, gait and visual disturbances, and increased BP after 5 days of initiation of sorafenib. There were no hemorrhagic, ischemic, or metastatic pathologic findings on his brain MRI. However, all his clinical signs and symptoms were consistent with PRES. His clinical symptoms were resolved after 5 days of discontinuation of sorafenib. ${ }^{11}$

Our case is the first pediatric case of a patient with FLT3-ITD-positive AML who developed PRES while receiving sorafenib treatment. The BP of our patient was above cutoff limits for hypertension at diagnosis of PRES, but returned to normal after the seizure. He did not require any antihypertensive medications. The clinical signs related to PRES were headache, hypertension, and a generalized tonic-clonic seizure. All of the clinical symptoms were resolved within 24 hours after discontinuation of sorafenib. During the treatment with cytarabine (IV and IT) and clofarabine, there were no symptoms related to PRES in our patient. When symptoms related to PRES appeared, the patient was only taking sorafenib. Furthermore, IT treatment was performed 20 days before the development of PRES. Therefore, sorafenib is likely the responsible agent for development of PRES in our patient.

The pathogenesis of PRES is not precisely known. It is known that the mechanism of PRES depends on failure of
A

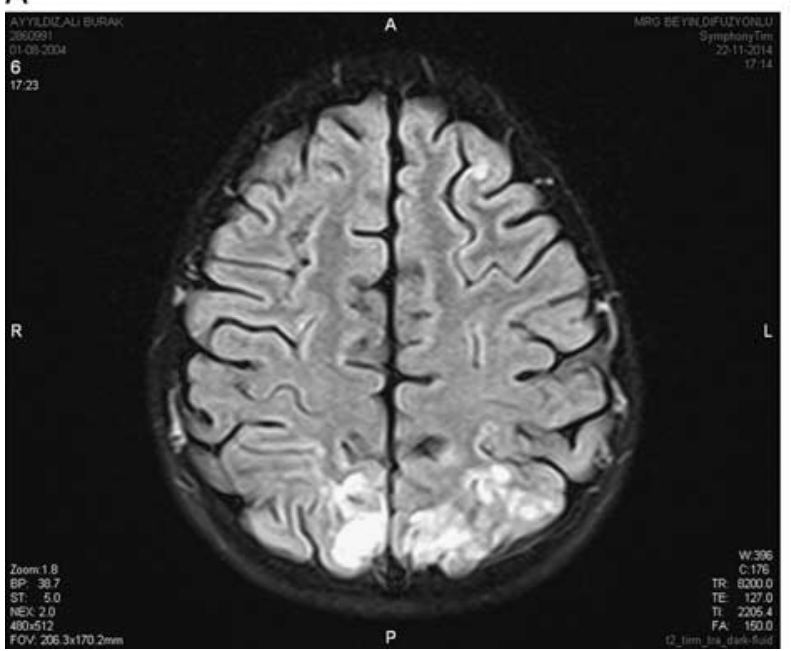

B

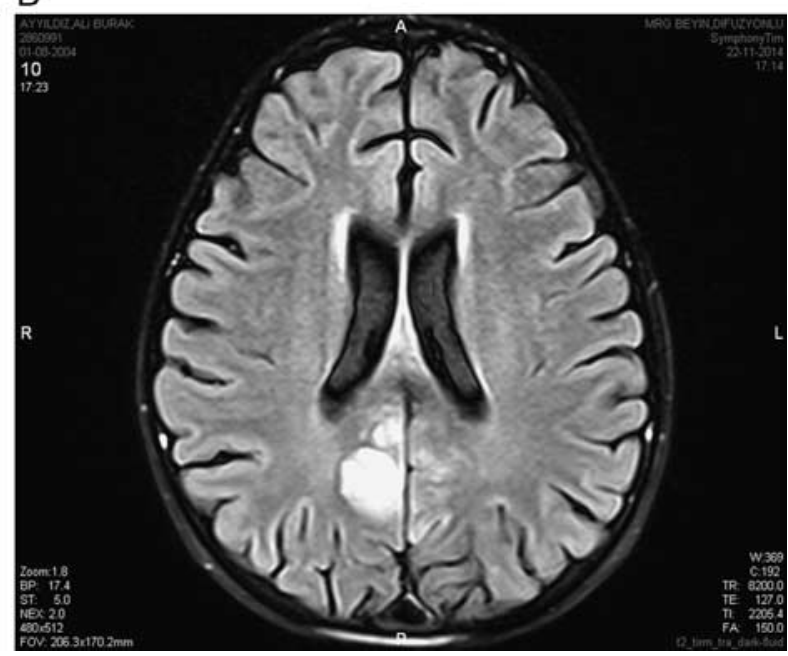

FIGURE 1. A, Brain MRI showing confluent hyperintense T2 signal involving subcortical white matter of the parietooccipital lobes consistent with PRES. B, Brain MRI showing confluent hyperintense T2 signal involving subcortical white matter of the parietooccipital lobes consistent with PRES. MRI indicates magnetic resonance imaging; PRES, posterior reversible encephalopathy syndrome. 
the blood-brain barrier to maintain the compartmentalization of intravascular fluid. This alteration of the bloodbrain barrier depends not only on the systematic BP but also on the integrity of the vascular endothelium, which is influenced by various medical conditions and by immunosuppressive and cytotoxic drugs. VEGF is a critical factor for vascular permeability. The disruption of the VEGF signaling pathway results in a decrease in nitric oxide bioavailability, suggesting that not only hypertension but also other pharmacodynamic effects of anti-VEGF agents could trigger the occurrence of PRES. ${ }^{7-11}$ Sorafenib is an inhibitor of proangiogenic receptor tyrosine kinases, such as VEGF receptor 2 and 3 and platelet-derived growth factor receptor $\beta$. It has an antiangiogenic activity and causes changes in the integrity of the vascular endothelium. ${ }^{3,4}$ As our patient had no hypertension before PRES, the antiangiogenic activity of sorafenib could be the cause for the development of PRES in our case.

In conclusion, PRES is a serious toxic, but manageable, side effect of sorafenib that should be considered by pediatric hematologists and oncologists using this drug for pediatric cases with FLT3-ITD-positive AML.

\section{REFERENCES}

1. Morris EB, Laningham FH, Sandlund JT, et al. Posterior reversible encephalopathy syndrome in children with cancer. Pediatr Blood Cancer. 2007;48:152-159.

2. Hasskarl J. Sorafenib: targeting multiple tyrosine kinases in cancer. Recent Results Cancer Res. 2014;201:145-164.

3. Knapper S. The clinical development of FLT3 inhibitors in acute myeloid leukemia. Expert Opin Investig Drugs. 2011;20: 1377-1395.
4. Swords R, Freeman C, Giles F. Targeting the FMS-like tyrosine kinase 3 in acute myeloid leukemia. Leukemia. 2012;26:2176-2185.

5. National High Blood Pressure Education Program Working Group on High Blood Pressure in Children and Adolescents. The fourth report on the diagnosis, evaluation, and treatment of high blood pressure in children and adolescents. Pediatrics. 2004;114(suppl 4th report):555-576.

6. Hinchey J, Chaves C, Appignani B, et al. A reversible posterior leukoencephalopathy syndrome. $N$ Engl J Med. 1996;334: 494-500.

7. Ozcan C, Wong SJ, Hari P. Reversible posterior leukoencephalopathy syndrome and bevacizumab. $N$ Engl $\mathrm{J}$ Med. 2006;354:980-982.

8. Govindarajan R, Adusumilli J, Baxter DL, et al. Reversible posterior leukoencephalopathy syndrome induced by RAF kinase inhibitor BAY 43-9006. J Clin Oncol. 2006;24:e48.

9. Martín G, Bellido L, Cruz JJ. Reversible posterior leukoencephalopathy syndrome induced by sunitinib. $J$ Clin Oncol. 2007;25:3559.

10. Saito B, Nakamaki $T$, Nakashima $H$, et al. Reversible posterior leukoencephalopathy syndrome after repeat intermediate-dose cytarabine chemotherapy in a patient with acute myeloid leukemia. Am J Hematol. 2007;82:304-306.

11. Dogan E, Aksoy S, Arslan C, et al. Probable sorafenib-induced reversible encephalopathy in a patient with hepatocellular carcinoma. Med Oncol. 2010;27:1436-1437.

12. Levy CF, Oo KZ, Fireman F, et al. Reversible posterior leukoencephalopathy syndrome in a child treated with bevacizumab. Pediatr Blood Cancer. 2009;52:669-671.

13. de Pasquale MD, Castellano A, de Sio L, et al. Bevacizumab in pediatric patients: how safe is it? Anticancer Res. 2011;31: 3953-3957.

14. Da Silva FMRS, Pego PMB, Vendrell MCH, et al. Posterior reversible encephalopathy syndrome and anti-angiogenic agents: a case report. Neuro-ophthalmology. 2011;35:32-37. 